\title{
Critical consideration of the Archives of Craniofacial Surgery journal homepage and tips for using its helpful features
}

\author{
Kang Young Choi \\ Department of Plastic and Reconstructive Surgery, School of Medicine, Kyungpook National University, Daegu, Korea
}

Archives of Craniofacial Surgery (Arch Craniofac Surg, ACFS) was launched in October 2000 as the Journal of the Korean Cleft Palate-Craniofacial Association, and since 2012 it has been published under its current name. ACFS is the official journal of the Korean Cleft Palate-Craniofacial Association, and it has published a total of 29 review articles, 364 original articles, 282 case reports, four ideas and innovations, eight images, seven letters, and two randomized controlled trials, for a total of 696 articles.

ACFS is a journal with an explicitly international scope that covers all surgical and medical fields associated with the craniofacial region, and it is indexed in PubMed, PubMed Central (PMC), Scopus, KoreaMed, KoMCI, Crossref, and Google Scholar [1,2].

In addition, the journal homepage, which has a full online submission system, offers various services and contains descriptions on how to carry out various tasks, ranging from the basic work involved in article creation to article submission. However, authors may be unfamiliar with its useful features, and some elements of the system may need modification.

\section{Article search}

The menu bar at the top of the main page of the homepage contains the following sections: "Home," "About," "Article Category," "Browse Articles," and "Author Information," making it

\section{Correspondence: Kang Young Choi}

Department of Plastic and Reconstructive Surgery, School of Medicine,

Kyungpook National University, 130 Dongdeok-ro, Jung-gu, Daegu 41944, Korea

E-mail: kychoi@knu.ac.kr

Received February 11, 2020 / Revised February 12, 2020 / Accepted February 13, 2020 possible for the user to intuitively access the necessary items by moving the mouse over these headings to reveal more detailed menus. The right side of the screen also contains the Search and Advanced Search menus, through which it is possible to find full articles published in ACFS according to detailed parameters. In approximately the middle of a vertical bar running down the right side of the main screen, various keywords are displayed in a white box, through which the full texts of articles associated with those keywords can be accessed with a single click (Figs. 1, 2). Free full-text PDFs are available for articles published since 2012, while abstracts are available for previously published articles. Additionally, related papers are displayed separately next to the abstract of each paper [3].

An issue currently facing ACFS is that full-text articles must be provided for pre-2012 papers for the journal to be truly open-access. This is especially important because the papers published before 2012 have been retrieved at least 300 times. Additionally, the accuracy of the article search menu should also be improved. The search results for "fracture" are relatively accurate, as shown in Figs. 1 and 2, but the search results for "Noma" are quite inaccurate, as shown in Fig. 3. Therefore, it is necessary to supplement the system to allow users to perform more accurate searches.

\section{Article writing}

When writing an article, authors should first read the manuscript preparation section in the Instructions for Authors subsection of the Author Information menu along the top of the 


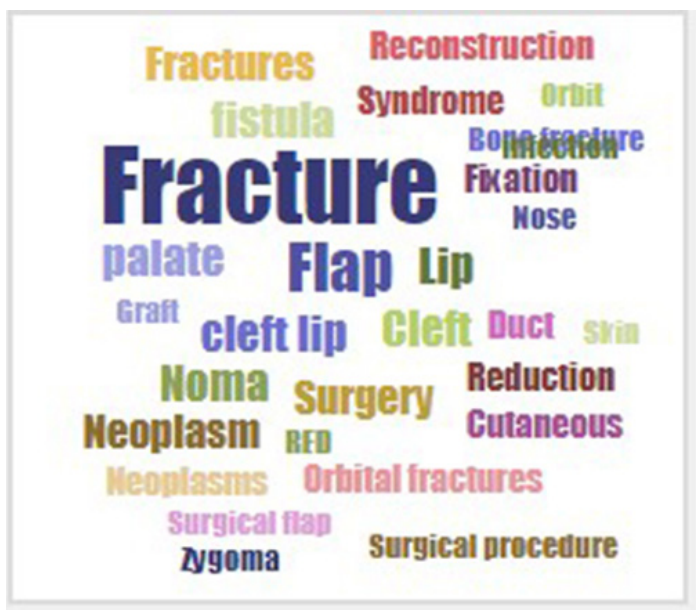

Fig. 1. Keyword box in the right middle corner of the homepage.

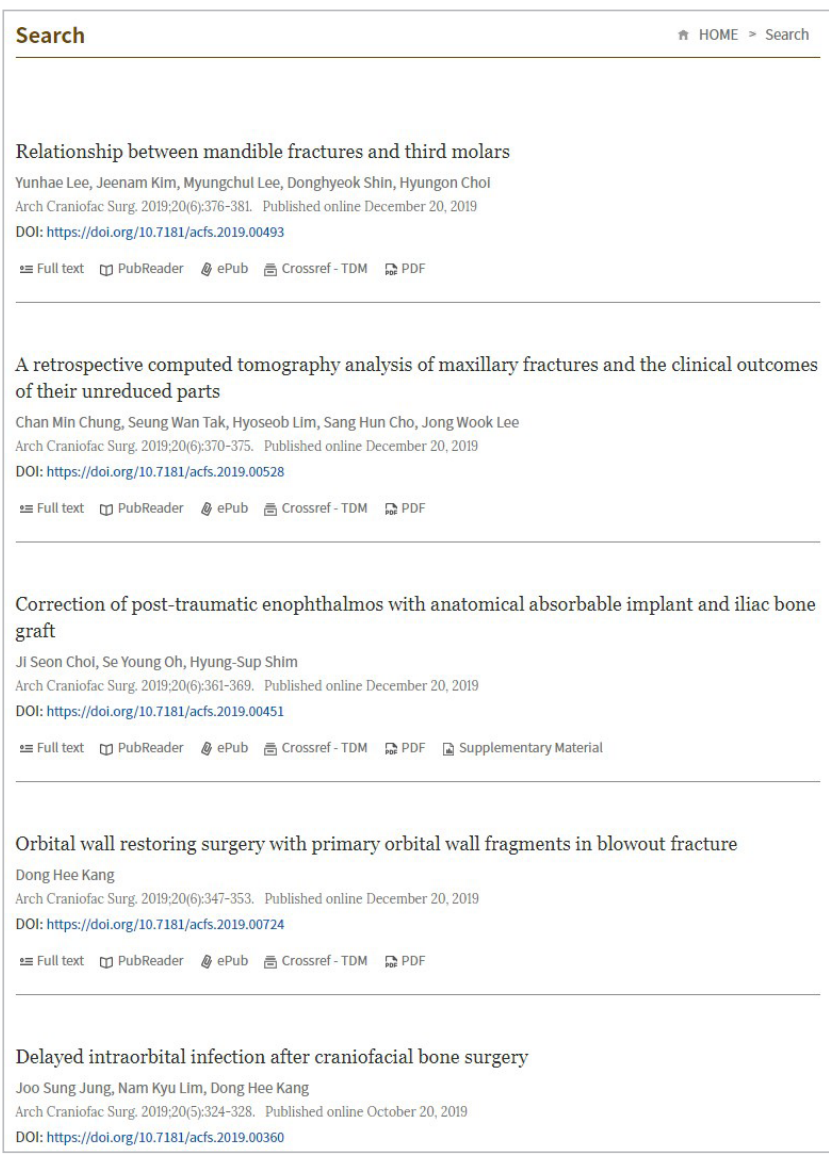

Fig. 2. The results obtained by clicking on "fracture" in the keyword box.

paper homepage. ACFS provides a detailed guideline for submission. The format is in accordance with the most commonly used format in medical journals, so authors should not experience any undue difficulties.

However, an additional piece of guidance for authors would be to make note of the format required depending on the type

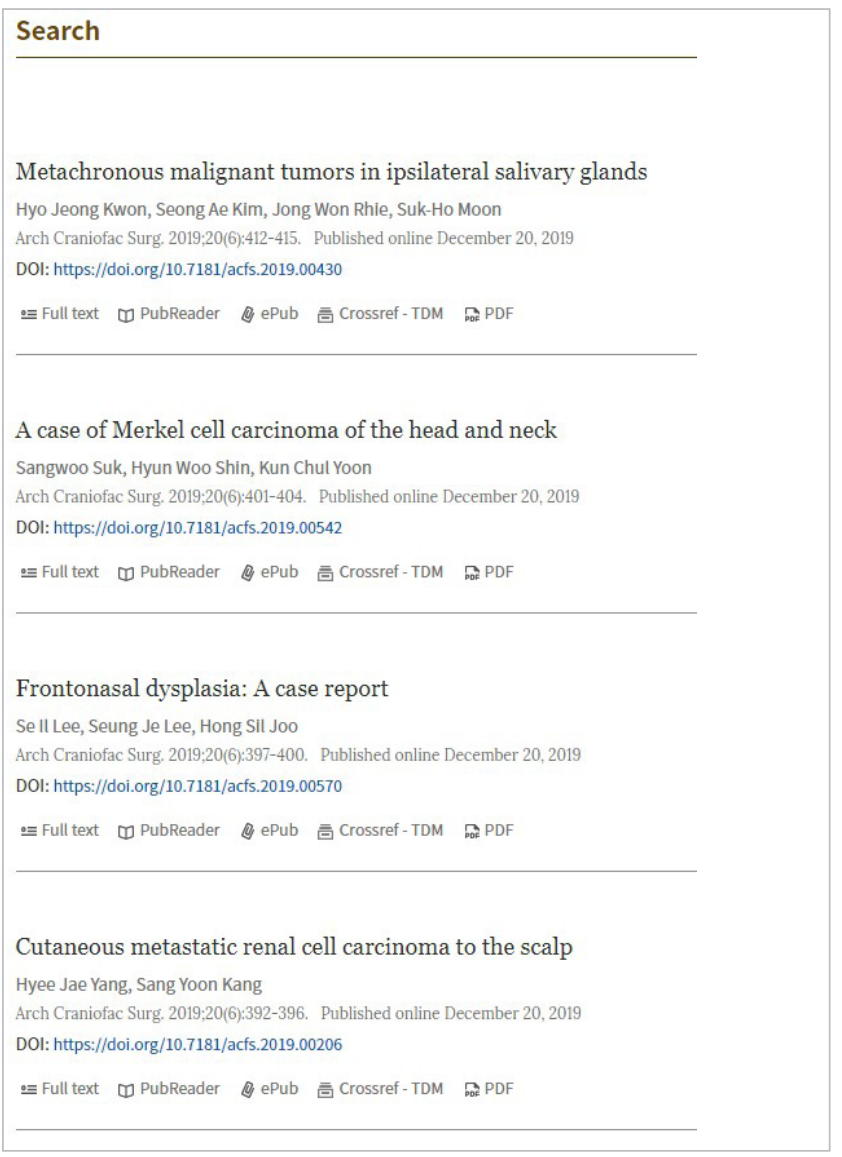

Fig. 3. The results obtained by clicking "Noma" on the keyword box.

of the submission-especially with regard to the tables, figures, and references-in order to avoid making mistakes later on in the writing process. It may be helpful to copy some of the examples shown for reference while writing the article.

In addition, for studies with a specific design, such as randomized controlled studies, studies of diagnostic accuracy, meta-analyses, observational studies, and non-randomized studies, it is recommended that authors follow the reporting guidelines listed in the section entitled "Reporting Guidelines for Specific Study Designs." This is a particular strength of ACFS and an area in which it is comparable to other leading journals.

\section{Article submission}

If the guidance presented above has been followed, the paper is now in line with the ACFS guidelines and only the submission process remains. The main page for article submissions can be accessed through the "E-submission" link in the Author Information menu along the top menu bar or by clicking the "ESUBMISSION" button in the middle of the vertical bar running down the right side of the homepage. Through this interface, an author can submit a manuscript after completing the regis- 


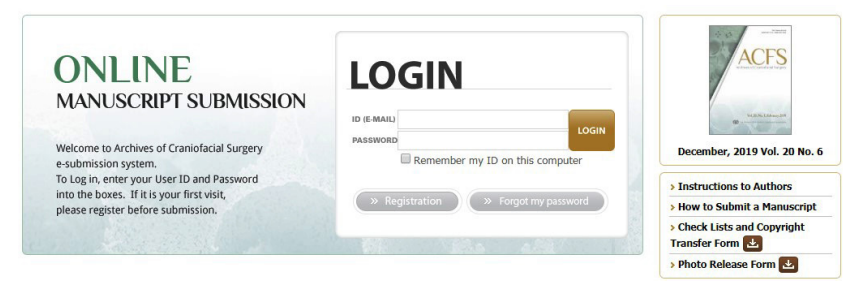

Fig. 4. Main page of the online submission system.

tration process described on the screen shown in Fig. 4. As an additional tip, the risk of errors can be reduced by preparing a file containing the author's English name, affiliation, address, email address, telephone, fax, and ORCID number in advance.

An issue here is that $A C F S$ requires several steps in this process. Of course, as with most journals, ACFS requires a title page, main body, table files, figure files, supplementary materials, and a copyright transfer agreement. However, modern documentation programs are of sufficiently high quality that, in my opinion, requiring all of these files after an article is accepted would reduce the burden on the author and facilitate the submission of articles. In fact, for Nature, the top journal in medicine, authors must only upload two files (cover letter and main text) and provide their information. Then, after a submission is accepted, it is quite convenient to upload the remaining files. Therefore, I think we need to discuss modifications to this part of the system.

After the submission is completed, an initial decision will normally be made within 3 weeks after the reviewers agree to review the article, and the reviewers' comments will then be sent to the corresponding author. Revised manuscripts must be submitted online by the corresponding author. The corresponding author must indicate the alterations that have been made in response to the referees' comments item by item in a response letter and in the originally submitted file with track changes. Failure to resubmit the revised manuscript within 8 weeks of the editorial decision is regarded as withdrawal.

ACFS offers an online manuscript submission system for rapid peer review and communication with authors. It also helps make the entire process of drafting and submitting a manuscript easier.

\section{NOTES}

\section{Conflict of interest}

$\mathrm{KYC}$, the co-editor of the Archives of Craniofacial Surgery, is the corresponding author of this article. However, he played no role whatsoever in the editorial evaluation of this article or the decision to publish it. Except for that, no potential conflict of interest relevant to this article was reported.

\section{ORCID}

Kang Young Choi https://orcid.org/0000-0002-7735-1939

\section{REFERENCES}

1. Kim KS. Korean Cleft Palate-Craniofacial Association: history and perspectives. Arch Craniofac Surg 2019;20:1-2.

2. Kim YH. Changes and directions of craniofacial surgery in Korea. Arch Craniofac Surg 2019;20:281-3.

3. Archives of Craniofacial Surgery (ACFS). Internet domain of ACFS [Internet]. Daegu: Korean Cleft Palate-Craniofacial Association [cited 2020 Apr 3]. Available from: https://e-acfs.org/. 